\title{
Concavity for nuclear bindings, thermodynamical functions and density functionals
}

\author{
B. R. Barrett \\ Physics Department, University of Arizona, \\ Tucson, AZ 85721, USA \\ B. G. Giraud \\ Institut de Physique Théorique, DSM, CE Saclay, \\ 91191 Gif-sur-Yvette, France \\ B. K. Jennings \\ TRIUMF, Vancouver BC, V6T2A3, Canada \\ N. P. Toberg \\ Polar Ocean Physics Group, Centre for Mathematical Sciences, \\ Cambridge, CB3 0WA, UK
}

November 16, 2018

\begin{abstract}
Sequences of experimental ground-state energies for both odd and even $A$ are mapped onto concave patterns cured from convexities due to pairing and/or shell effects. The same patterns, completed by a list of excitation energies, give numerical estimates of the grand potential $\Omega(\beta, \mu)$ for a mixture of nuclei at low or moderate temperatures $T=\beta^{-1}$ and at many chemical potentials $\mu$. The average nucleon number $\langle\mathbf{A}\rangle(\beta, \mu)$ then becomes a continuous variable, allowing extrapolations towards nuclear masses closer to drip lines. We study the possible concavity of several thermodynamical functions, such as the free energy and the average energy, as functions of $\langle\mathbf{A}\rangle$. Concavity, which always occurs for the free energy and is usually present for the average energy, allows easy interpolations and extrapolations providing upper and lower bounds, respectively, to binding energies. Such bounds define an error bar for the prediction of binding energies. Finally we show how concavity and universality are related in the theory of the nuclear density functional.
\end{abstract}

PACS: 21.10.Dr, 21.10.-k, 21.60.-n, 24.10.Pa

\section{Introduction}

The observation of a valley of stability and the search for mass formulae belong to the oldest subjects studied in nuclear physics 1] and continue to be of great 
interest today [2]. Given the neutron and proton numbers $N$ and $Z$ as independent variables and the corresponding atomic number, $A \equiv N+Z$, terms such as volume energy $\propto A$, surface tension $\propto A^{\frac{2}{3}}$, Coulomb energy $\propto Z(Z-1) / A^{1 / 3}$, symmetry energy $\propto(N-Z)^{2} / A$, etc., flourish in the literature, and a great deal of attention has been dedicated to the consideration of finer corrections, such as, for instance, terms $s(N, Z)$ and $p(N, Z)$ that account for shell and pairing effects, respectively, and further correlations. This work is motivated by the observation that the dominant terms, such as $\propto A, \propto Z(Z-1)$ and $\propto(N-Z)^{2}$, define a notoriously concave energy surface.

Upper and lower bounds to nuclear binding energies can be deduced from such a concavity, provided that deviations from concavity, possibly induced by subdominant terms like $\propto A^{\frac{2}{3}}, s(N, Z), p(N, Z)$, etc., can be corrected. For the sake of simplicity this paper first considers only sequences of isotopes and, thus, takes advantage of concavity with respect to $N$ only; $Z$ is frozen. In Section 2 we begin with a theory at zero temperature and show how elementary, invertible transformations of data can generate truly concave patterns. This is obtained by an analysis of the table of second differences between binding energies, then by a removal of the pairing energy, and finally by an ad hoc, but minimal, parabolic term added to the nuclear energies, if necessary.

Since concavity is also a property of several thermodynamical functions, an extension of the zero temperature analysis to finite temperatures is in order. In Section 3 we discuss properties of that grand potential, $\Omega(\beta, \mu)$, which can be deduced from the experimental data after their tuning. Other thermodynamical functions are also considered, and their concavity is tested. Bounds are found, and an error bar for predictions is estimated.

A generalization to concavity with respect to both $N$ and $Z$ is briefly studied in Section 4. An additional motivation for our investigation of concavity properties is the need, in density functional theories, of concavity, if the universality [3] of a density functional must be obtained. A solution will be shown.

Finally, a discussion and conclusion are given in Section 5.

\section{Concavity for experimental ground-state en- ergies}

Our argument is best illustrated numerically, by using a sequence of isotopic ground-state binding energies, $-E_{A}$. We choose to work with the tin isotopes $(\mathrm{Sn})$, because they provide a large number of known isotopes for testing our method. In addition, in Section 3, we will extend our approach to finite temperatures using a grand potential, which requires that a sufficiently large number of excited states are also known. For instance, a table of 25 ground-state bindings for the tin isotopes from ${ }^{110} \mathrm{Sn}$ to ${ }^{134} \mathrm{Sn}$ reads, in $\mathrm{keV}$, \{ 934571, 942744, 953531, 961274, 971574, 979120, 988684, 995627, 1004954, 1011438 , 1020546, 1026716, 1035529, 1041475, 1049963, 1055696, 1063889, 1069439, 1077345, 1082676, 1090293, 1095540, 1102851, 1105320, 1109239\}. 
Despite a standard linear trend because of a "not too much fluctuating average energy per nucleon", this list is not making a smooth pattern, even less of a concave one. The full line in Fig. 1, where, for graphical convenience, we have added to each $E_{A}$ a constant term, $115 \mathrm{MeV}$ and a linear term, $7.5 \times A$ $\mathrm{MeV}$, shows the amount of irregularity in the pattern. The main source of irregularity is, obviously, the pairing effect. If it can be removed, concavity emerges. Concavity for a sequence of isotones is not a surprise, because of the $Z(Z-1)$ nature of the Coulomb term. For a sequence of isotopes, however, there is no such obviously quadratic term available, notwithstanding the empirical modelization of a symmetry energy $\propto(N-Z)^{2}$. Our choice of Sn, because of its long isotope sequence, illustrates this concavity more dramatically.

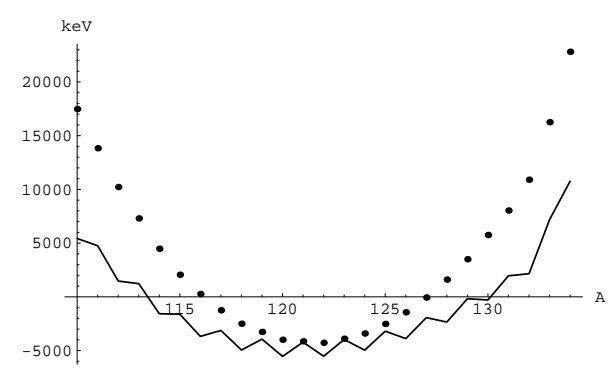

Figure 1: Sn isotope energies: irregular line joining raw experimental groundstate energies $E_{A}+7500 A+115000$; pairing and parabolic corrections give the nonconnected dots.

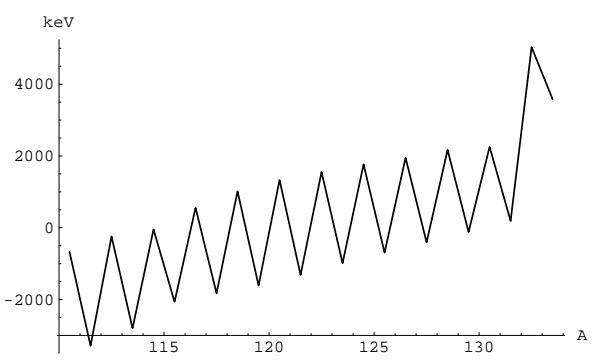

Figure 2: Staggering effect, seen from first differences $D=E_{A+1}-E_{A}+7500$.

Most energies stated in this paper are in units of keV. Our data come from Ref. [4]. See also [5, 6, 7]. These sources usually quote the binding energy per nucleon instead of the total binding energy itself and such values per nucleon are given to varying numbers of significant figures, from six to seven for the $\mathrm{Sn}$ isotopes 4 that we consider. Consequently, we quote and use all our binding energies to six or seven significant figures. Our values are generally accurate to the order of two to three $\mathrm{keV}$ near the center of the sequence, and tens of $\mathrm{keV}$ at both ends for the Sn isotopes. See, in particular, 4 for error bars.

The staggering effect is clear from the 24 first differences, $E_{A+1}-E_{A}+7500$, 
$\{-673,-3287,-243,-2800,-46,-2064,557,-1827,1016,-1608,1330,-1313,1554$, -988, 1767, -693, 1950, -406, 2169, -117, 2253, 189, 5031, 3581\}, see Fig. 2.

The list of 23 second differences (SDs), $S D=E_{A+1}-2 E_{A}+E_{A-1}$, $\{-2614,3044,-2557,2754,-2018,2621,-2384,2843,-2624,2938,-2643,2867$, $-2542,2755,-2460,2643,-2356,2575,-2286,2370,-2064,4842,-1450\}$, is insensitive to the constant and linear terms we used for graphical convenience. It gives estimates of the "curvatures" of the pattern. It turns out to be far from containing only positive numbers. The nonconnected points shown in Fig. 3 represent this pattern of SDs. A systematic oscillation, reflecting the staggering effect, is found. Alternating signs are obviously due to the gains of binding for even Sn nuclei because of pairing. The oscillation between SDs centered at odd and even nuclei has, roughly speaking, a constant amplitude. Notice, however, the maximum in the list, 4842 , due to the shell closure at ${ }^{132} \mathrm{Sn}$.

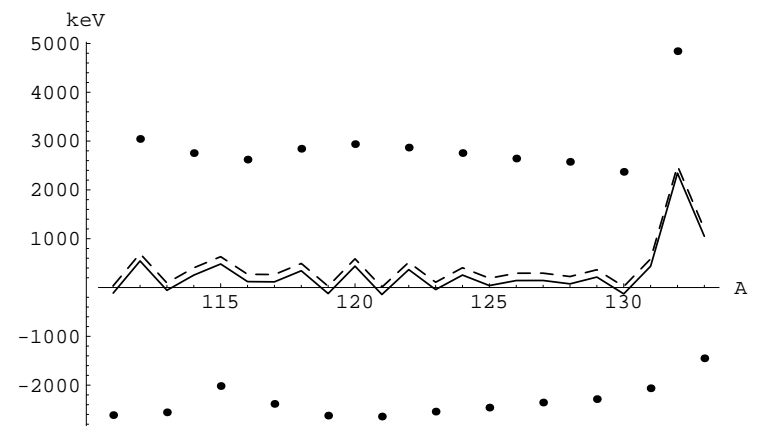

Figure 3: Second differences $S D=E_{A+1}-2 E_{A}+E_{A-1}$. Dots, raw data; solid line, result of the pairing correction; dashed line, final result after the parabolic correction.

Add now to each even nucleus energy a fixed number, for example $p(N, Z)=$ $1250 \mathrm{keV}$, to tentatively suppress the increase of binding due to pairing. The resulting list of SDs is attenuated by an amount equal to $\pm 2 p$, as illustrated by the full line in Fig. 3. The attenuated list reads, $\{-114,544,-57,254,482,121,116,343,-124,438,-143,367,-42,255,40,143$, $144,75,214,-130,436,2342,1050\}$. All numbers are now significantly smaller than their partners in the previous list of SDs, except for the smaller, but still large maximum at ${ }^{132} \mathrm{Sn}$. This maximum is positive, however, and causes no difficulty for concavity. The interesting point is rather the most negative number in the list, namely $-143 \mathrm{keV}$. All negative curvatures can be converted into positive ones if we add to every energy an artificial, parabolic correction, $P \times(A-122)^{2}$, with $P=75 \mathrm{keV}$. Incidentally, the lowest point, $A_{0}=122$, of the "added parabola" is arbitrary, because SDs will increase by just a constant, namely twice the coefficient $P$ of the $A^{2}$ term. After this $2 P=150 \mathrm{keV}$ shift, the whole sequence of SDs becomes positive,

$\{36,694,93,404,632,271,266,493,26,588,7,517,108,405,190,293,294$, 225, 364, 20, 586, 2492, 1200\}, see the dashed line shown in Fig. 3. 
In short, a "concavity ensuring" manipulation for the isotope energies consists in replacing each energy $E_{A}$ by $E_{A}^{\prime}=E_{A}+p \times \operatorname{Mod}[A+1,2]+P \times(A-$ $\left.A_{0}\right)^{2}+115000+7500 A$. (We recall that the terms which we have added are here just for graphical, and later, numerical convenience; they do not influence the theory.) With $p=1250, P=75$ and $A_{0}=122$, the list of such tuned energies $E_{A}^{\prime}$ (shown by the nonconnected dots in Fig. 1) reads,

$\{17479,13831,10219,7301,4476,2055,266,-1252,-2504,-3263,-3996,-4141$, $-4279,-3900,-3413,-2521,-1439,-64,1605,3499,5757,8035,10899,16255$, $22811\}$. The choice of the two parameters, $\mathrm{p}=1250 \mathrm{keV}$ and $\mathrm{P}=75 \mathrm{keV}$, is empirical: one looks for a pairing correction leading to a modest, if not minimal, parabolic correction inducing concavity. Other choices for $\{p, P\}$ are possible, but, obviously, within a small range around 1250 and 75 . Furthermore, such parameters must be readjusted for different regions of the table of nuclei, but it is again obvious that readjustments will be moderate; for instance, the order of magnitude for pairing will always be around $\sim 1.0$ to $\sim 1.5 \mathrm{MeV}$. Analyzing short sequences obviously leaves fewer negative SDs to be compensated by the artificial, parabolic term, and, hence should induce smaller values of $P$. For this reason, we would expect short sequences to give often better extrapolations.

Once concavity is obtained, it is straightforward that extrapolations from two points on the concave pattern allow predictions of lower bounds to nuclear energies and interpolations give upper bounds. Then, from such bounds for energies $E^{\prime}$, one recovers bounds, of strictly the same quality, for the physical energies $E$. This obtains by subtracting from each $E^{\prime}$ bound its "tuning term".

Assume now that ${ }^{110} \mathrm{Sn}$ were unknown and one had done a brute force extrapolation, $2 E_{111}-E_{112}=-931957$. Compared with $E_{110}=-934571$, this prediction underbinds ${ }^{110} \mathrm{Sn}$ by $2614 \mathrm{keV}$. Consider rather $2 E_{111}^{\prime}-E_{112}^{\prime}=17443$, to be compared with $E_{110}^{\prime}=17479$. A slight overbinding, by $36 \mathrm{keV}$, is found. Although this small error is likely accidental, it is clear that systematic lower bounds will be found. Naturally, once a value for $E_{A}^{\prime}$ is predicted, one recovers as good an estimate for $E_{A}$ after removing the "concavity manipulation terms", which are known explicitly. In the present case, the tuning of the data added 952050 to $E_{110}$. The same 952050 must be subtracted from that value, $2 E_{111}^{\prime}-E_{112}^{\prime}=17443$, extrapolated from the concave pattern, yielding a final result of -934607 , to be compared with $E_{110}=-934571$, which obviously exhibits the same slight overbinding, $36 \mathrm{keV}$. Based on the data in 4, the experimentally measured energy for ${ }^{110} \mathrm{Sn}$ has an error bar $\pm 14 \mathrm{keV}$; hence, in this case, the experimental error bar and the uncertainty in our theoretical value have the same order of magnitude.

If ${ }^{132} \mathrm{Sn}$ were unknown, the brute force extrapolation gives, $2 E_{131}-E_{130}=$ -1100787 , to be compared with $E_{132}=-1102851$, showing an underbinding equal to $2064 \mathrm{keV}$, clearly failing to reproduce the shell closure effect. Now, from the concave pattern, we obtain $2 E_{131}^{\prime}-E_{130}^{\prime}=10313$, to be compared with $E_{132}^{\prime}=10899$, producing an overbinding by $586 \mathrm{keV}$. The same overbinding is obviously found if one subtracts from $2 E_{131}^{\prime}-E_{130}^{\prime}=10313$, the tuning difference, $E_{132}^{\prime}-E_{132}=1113750$, with the result, -1103437 , to be compared with $E_{132}=-1102851$. See Fig. 4, which also illustrates an extrapolation using 
$E_{133}$ and $E_{134}$ and an interpolation using $E_{131}$ and $E_{133}$.
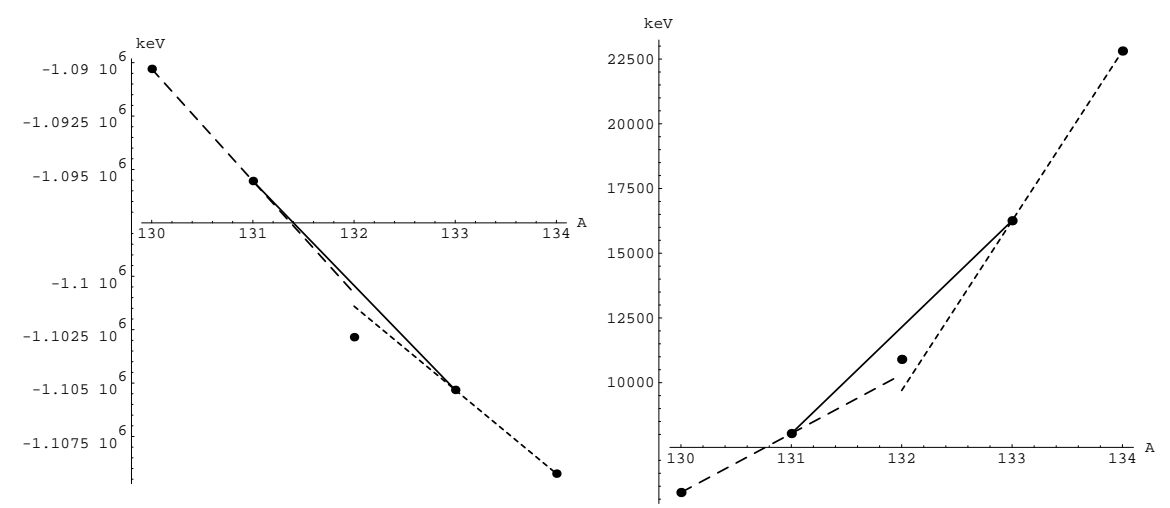

Figure 4: Left, raw energies: full line, interpolation from ${ }^{131} \mathrm{Sn}$ and ${ }^{133} \mathrm{Sn}$; long dashes, extrapolation from ${ }^{130} \mathrm{Sn}$ and ${ }^{131} \mathrm{Sn}$; short dashes, extrapolation from ${ }^{134} \mathrm{Sn}$ and ${ }^{133} \mathrm{Sn}$. Dots represent the experimental energies $E_{A}$. Right: same inter- and extrapolations, concave data.

Let us now also compare for an odd rather than even isotope, e.g., ${ }^{117} \mathrm{Sn}$, two extrapolations and one interpolation from raw data and concave data, respectively. With our choice of parameters, the tuning difference, $E_{117}^{\prime}-E_{117}$, is 994375 , with $E_{117}=-995627$. Extrapolations from lower masses give, $2 E_{116}-$ $E_{115}=-998248$, and $2 E_{116}^{\prime}-E_{115}^{\prime}-994375=-995898$, hence, overbindings of 2671 and 271, respectively. Extrapolations from higher masses give, $2 E_{118}-E_{119}=-998470$, and $2 E_{118}^{\prime}-E_{119}^{\prime}-994375=-996120$, leading to overbindings of 2843 and 493, respectively. Interpolations from the nearest nuclei give, $\left(E_{116}+E_{118}\right) / 2=-996819$, and $\left(E_{116}^{\prime}+E_{118}^{\prime}\right) / 2-994375=-995494$, with now overbinding of 1192 and underbinding of 133, respectively. The poor results from raw data stress the importance of pairing corrections. The much better results from the concave pattern do take this pairing into account, but, furthermore, they again make it trivial that extrapolations give overbindings, while interpolations give underbindings.

For the sake of completeness, we now consider a case with a two-step extrapolation. If the tuned pattern followed a perfect parabolic form, $E_{A}^{\prime}=$ $a A^{2}+b A+c$, the formula, $E_{A+2}^{\prime}-3 E_{A}^{\prime}+2 E_{A-1}^{\prime}=6 a$, predicts, for the lower bound, an error which is a little larger than desirable. This is because of the coefficient, 6 , and the fact that the "curvature parameter", $a$, has here an order of magnitude in the few hundred $\mathrm{keV}$ range, see those residual second differences used for the dashed curve in Fig. 3. This indicates that further stages of the theory, with polynoms at least, are worthy of consideration. However, to remain within the "linear" scope of the present paper, we give the results obtained for ${ }^{135} \mathrm{Sn},-1113208$, and ${ }^{136} \mathrm{Sn},-1115027$, when we use the lower bound estimates from the formula, $3 E_{A}^{\prime}-2 E_{A-1}^{\prime}$. From Ref. [4, the predictions, which are based on neighbor nuclei with the same $Z$ and $N$ parities, are -1111320 and 
-1115064 , respectively, with error bars of $\sim \pm .5 \mathrm{MeV}$. We do find a lower bound for ${ }^{135} \mathrm{Sn}$, with apparently a severe, but mostly expected, error of a couple of MeVs. For ${ }^{136} \mathrm{Sn}$, we apparently fail to generate a lower bound, but by so small a difference that our results stay well inside the error bars.

To summarize Section 2, when concavity occurs, one concludes that extrapolations from two points on the concave pattern allow predictions of lower bounds to nuclear energies. In the same way, interpolations provide upper bounds. The quality of such "concavity bounds" from zero temperature data is found to be good; interpolations and extrapolations from the raw, nonconcave pattern, are clearly less satisfactory. But there is a more profound reason why a concave pattern is necessary. Indeed, several thermodynamical functions, governed by theorems proving their concavity, have a notoriously singular limit at zero temperature: they become nonanalytical and are just piecewise continuous. Their limit plots are made of segments; derivatives are discontinuous at turning points. Because of the staggering effect, the concave envelope of the raw pattern of $E_{A}$ 's would contain only the even isotopes. Concavity is, thus, necessary for a theory that must accommodate both odd and even nuclei. Therefore, the thermodynamical functions studied in Section 3 preferably use concave energies $E_{A}^{\prime}$ and the corresponding excited state energies $E_{n A}^{\prime}$.

\section{Concavity with thermodynamical functions}

Consider the particle number operator $\mathbf{A}$ and a familiar nuclear Hamiltonian $\mathbf{H}=\sum_{i=1}^{A} t_{i}+\sum_{i>j=1}^{A} v_{i j}$, where $A, t$ and $v$ are the mass number, one-body kinetic energy and two-body interaction, respectively. Nuclear data tables [8, 9] give precise values for a large number of lowest-lying eigenvalues $E_{n A}$ of $\mathbf{H}$, for many nuclei. One may, thus, reasonably estimate the grand partition function,

$$
\mathcal{Z}(\mu, \beta)=\operatorname{Tr} \exp [\beta(\mu \mathbf{A}-\mathbf{H})]=\sum_{n A}\left(2 j_{n A}+1\right) \exp \left[\beta\left(\mu A-E_{n A}\right)\right],
$$

provided that i) the temperature, $T=\beta^{-1}$, is low enough to allow a truncation of the spectrum to include only those states provided by the tables and ii) the chemical potential, $\mu$, selects mainly those nuclei in which we are interested. Let \langle\rangle denote, as usual, a statistical average. The (equilibrium!) density operator in Fock space, $\rho=\mathcal{Z}^{-1} \exp [\beta(\mu \mathbf{A}-\mathbf{H})]$, ensures that the following grand potential, $\Omega(\mu, \beta)=\langle(\mathbf{H}-\mu \mathbf{A})\rangle-T \mathbf{S}$, is minimal in the space of many-body density matrices with unit trace, since, by definition, $\langle\mathbf{A}\rangle=\operatorname{Tr} \rho \mathbf{A},\langle\mathbf{H}\rangle=$ $\operatorname{Tr} \rho \mathbf{H}$, with the entropy, $\mathbf{S}=-\operatorname{Tr}(\rho \log \rho)$.

We shall rather use $(-\Omega)$ in the following, to make upcoming proofs of concavity slightly easier. This grand potential also reads,

$$
-\Omega(\mu, \beta)=\beta^{-1} \ln \mathcal{Z}=\beta^{-1} \ln \left\{\sum_{n A}\left(2 j_{n A}+1\right) \exp \left[\beta\left(\mu A-E_{n A}\right)\right]\right\},
$$


Simple manipulations then give the relevant statistical averages \langle\rangle of particle numbers and energies, together with their derivatives and fluctuations,

$$
\partial(-\Omega) / \partial \mu=\langle\mathbf{A}\rangle=\sum_{A} A p_{A}, \quad p_{A}=\mathcal{Z}^{-1} \sum_{n}\left(2 j_{n A}+1\right) \exp \left[\beta\left(\mu A-E_{n A}\right)\right],
$$

and

$$
\partial^{2}(-\Omega) / \partial \mu^{2}=\partial\langle\mathbf{A}\rangle / \partial \mu=\beta\left(\left\langle\mathbf{A}^{2}\right\rangle-\langle\mathbf{A}\rangle^{2}\right),
$$

then

$$
\partial(-\Omega) / \partial T=\mathbf{S}=\ln \mathcal{Z}-\beta\langle(\mu \mathbf{A}-\mathbf{H})\rangle,
$$

or as well,

$$
\langle\mathbf{H}\rangle=\mathcal{Z}^{-1} \sum_{n A}\left(2 j_{n A}+1\right) E_{n A} \exp \left[\beta\left(\mu A-E_{n A}\right)\right]
$$

Furthermore,

$$
\partial^{2}(-\Omega) / \partial T^{2}=\beta^{3}\left[\left\langle(\mu \mathbf{A}-\mathbf{H})^{2}\right\rangle-\langle(\mu \mathbf{A}-\mathbf{H})\rangle^{2}\right],
$$

and

$$
\partial^{2}(-\Omega) /(\partial \mu \partial T)=-\beta^{2}[\langle\mathbf{A}(\mu \mathbf{A}-\mathbf{H})\rangle-\langle\mathbf{A}\rangle\langle(\mu \mathbf{A}-\mathbf{H})\rangle] .
$$

For our investigations, we will freeze $\beta$ as real and consider functions of a real $\mu$. It is then well known that $(-\Omega)$ is a concave function of $\mu$ and $T$. In turn, the double Legendre transform, with respect to both $\mu$ and $T$,

$$
\mu \partial(-\Omega) / \partial \mu+T \partial(-\Omega) / \partial T+\Omega=\langle\mathbf{H}\rangle,
$$

shows that $\langle\mathbf{H}\rangle$ is a concave function of both $\langle\mathbf{A}\rangle$ and $\mathbf{S}$, the conjugate variables of $\mu$ and $T$, respectively.

In the following, we do not perform the full, double Legendre transform. We rather retain an intermediate representation, with $\langle\mathbf{A}\rangle$ and either $T$ or $\beta$. We stay with real variables and functions. We stress that, while $\mathbf{A}$ has a discrete spectrum, $\langle\mathbf{A}\rangle$ is continuous, a monotonically increasing function of $\mu$, smooth provided $\beta$ is finite. The monotonicity results from Eq. (4). Actually, at low temperatures, strong derivatives signal the onset of discrete jumps due to the integer spectrum of $\mathbf{A}$, but we may stay away from this "jumpy" regime in the following, at least temporarily. Anyhow, at any fixed, finite $\beta$, the smoothness and monotonicity of $\langle\mathbf{A}\rangle$ with respect to $\mu$ allows a reasonably easy numerical calculation of the inverse function $\mu(\langle\mathbf{A}\rangle)$. Thus, a main argument of this Section is that at fixed temperatures we will use $\langle\mathbf{A}\rangle$ as a continuous variable and attempt extrapolations towards unknown nuclei.

For this, given a value of $T$, we keep track of $\langle\mathbf{A}\rangle$ and $\langle\mathbf{H}\rangle$ as functions of $\mu$. Since the functional inversion from $\langle\mathbf{A}\rangle(\mu)$ to $\mu(\langle\mathbf{A}\rangle)$ is reasonably easy, we can plot $\langle\mathbf{H}\rangle$ in terms of $\langle\mathbf{A}\rangle$ and attempt an extrapolation for further values of $\langle\mathbf{A}\rangle$. This extrapolation can be considered as a "candidate" for a mass formula, at that finite temperature $T$. 
According to Eq. (77), the average, constrained energy, $\langle(\mathbf{H}-\mu \mathbf{A})\rangle$, is a monotonically decreasing function of $\beta$. Furthermore, at least for negative chemical potentials $\mu$, and, more generally, if $A$ has an upper bound, the operator, $\mathbf{H}-\mu \mathbf{A}$, is bounded from below. Therefore, there is a convergence of the process consisting in i) extrapolating with respect to $\mu$ both $\langle(\mathbf{H}-\mu \mathbf{A})\rangle$ and $\langle\mathbf{A}\rangle$ for fixed values of $\beta$, then ii) eliminating $\mu$ to generate the $\beta$-parametrized "mass formula" $\langle\mathbf{H}\rangle(\langle\mathbf{A}\rangle, \beta)$, and finally iii) considering the limit of this mass formula when $\beta \rightarrow+\infty$. Alternately, it is equivalent, and maybe more efficient, to first eliminate $\mu$ and then extrapolate the "mass formula" $\langle\mathbf{H}\rangle(\langle\mathbf{A}\rangle, \beta)$, first with respect to $\langle\mathbf{A}\rangle$, then with respect to $\beta$.

Is there concavity in this intermediate representation? Clearly, a simple Legendre transform of $(-\Omega)$, with respect to $\mu$ only, returns a free energy, $\mathbf{F}=\langle\mathbf{H}\rangle-T \mathbf{S}$, as a concave function of $\langle\mathbf{A}\rangle$ and $T$. If $T$ is low enough to allow the product $T \mathbf{S}$ to be neglected, then, at fixed $T$, one may accept that $\langle\mathbf{H}\rangle$ is an "almost" concave function of $\langle\mathbf{A}\rangle$. This assumption will be tested by the numerical results which follow. Incidentally, a straightforward calculation of $A^{\prime \prime}(\langle\mathbf{A}\rangle, \beta) \equiv$ $\partial^{2}\langle\mathbf{H}\rangle /(\partial\langle\mathbf{A}\rangle)^{2}$ yields, $A^{\prime \prime} \propto\left(\left\langle\mathbf{A}^{2}\right\rangle-\langle\mathbf{A}\rangle^{2}\right)\left\langle\mathbf{A}^{2} \mathbf{H}\right\rangle+\left(\langle\mathbf{A}\rangle\left\langle\mathbf{A}^{2}\right\rangle-\left\langle\mathbf{A}^{3}\right\rangle\right)\langle\mathbf{A} \mathbf{H}\rangle+$ $\left(\langle\mathbf{A}\rangle\left\langle\mathbf{A}^{3}\right\rangle-\left\langle\mathbf{A}^{2}\right\rangle^{2}\right)\langle\mathbf{H}\rangle$, with a positive factor, $\left(\left\langle\mathbf{A}^{2}\right\rangle-\langle\mathbf{A}\rangle^{2}\right)^{-3}$. More simply,

$$
\frac{\partial^{2}\langle\mathbf{H}\rangle}{\partial\langle\mathbf{A}\rangle^{2}}(\langle\mathbf{A}\rangle, \beta) \propto\left\langle(\Delta \mathbf{A})^{2}\right\rangle\left\langle(\Delta \mathbf{A})^{2} \Delta \mathbf{H}\right\rangle-\left\langle(\Delta \mathbf{A})^{3}\right\rangle\langle\Delta \mathbf{A} \Delta \mathbf{H}\rangle,
$$

if one uses the centered operators, $\Delta \mathbf{A}=\mathbf{A}-\langle\mathbf{A}\rangle$ and $\Delta \mathbf{H}=\mathbf{H}-\langle\mathbf{H}\rangle$. From Eq. (10), concavity for $\langle\mathbf{H}\rangle$ is unclear; we shall have to test it numerically.

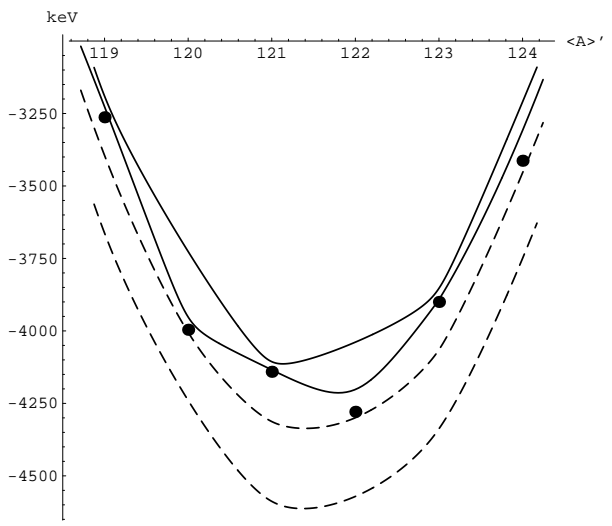

Figure 5: Energy $\langle\mathbf{H}\rangle^{\prime}$ (full curves) and free energy $\mathbf{F}^{\prime}$ (dashed ones) as functions of $\langle\mathbf{A}\rangle^{\prime}$, calculated from concavity tuned energies $E_{n A}^{\prime}$. Dots represent tuned, ground-state energies, $E_{0 A}^{\prime} \equiv E_{A}^{\prime}$, already used in Fig. 1 and the rhs part of Fig. 4. Upper full and lower dashed curves, $T=150 \mathrm{keV}$. Lower full and upper dashed curves, $T=60 \mathrm{keV}$. Notice how the full curves turn out to be concave.

The results, which follow, use [8, 9] for the excited-state energies of the $\mathrm{Sn}$ isotopes. Using the first 10 levels of ${ }^{110} \mathrm{Sn}$ to ${ }^{132} \mathrm{Sn}$, hence a maximum 
excitation of, typically, $\sim 1.2 \mathrm{MeV}$ for odd isotopes and twice as much for even ones, we calculate $\mathcal{Z}$, see Eq. (1). For those rare cases, where the spin $j_{n A}$ is ambiguous, we choose the lowest of the suggested spins. If the spin is completely unknown, we set it to be either 0 or $1 / 2$, according to $A$. These tactics minimize the statistical influence of such rare cases. The highest excited levels that we use have an excitation energy of order a few $\mathrm{MeV}$; hence, we restrict our thermodynamical analysis to temperatures of order $\sim 500 \mathrm{keV}$ at most. It should be noted that we use the concavity tuned energies, $E_{n A}^{\prime}=$ $E_{n A}+115000+7500 A+1250 \operatorname{Mod}[A+1,2]+75(A-122)^{2}$. Consequently, all our calculated quantities, $\langle\mathbf{A}\rangle^{\prime}, \mathbf{S}^{\prime},\langle\mathbf{H}\rangle^{\prime}$, etc., derived from Eqs. (3), (5), and (6), for instance, carry a prime superscript. We want to stress that the spurious exponential factor created by the constant, 115000 , appears in the same way in numerators and denominators and, hence, cancels out; it only helps the graphics and numerics. Similarly, the linear term, $7500 \mathrm{~A}$, means but a change of reference for the chemical potential. Only the pairing and parabolic tuning terms make the primed quantities truly distinct from those "raw", unprimed ones, obtained with the untuned energies $E_{n A}$.

As an example of our results we choose for Fig. 5 the sequence of six isotopes from ${ }^{119} \mathrm{Sn}$ to ${ }^{124} \mathrm{Sn}$, because of its still severely contrasted list of second differences, $\{588,7,517,108\}$, after tuning. This produces a serious geometrical constraint on the plots of $\langle\mathbf{H}\rangle^{\prime}$ and $\mathbf{F}^{\prime}$, as they reach their zero temperature limit, and better validates our approach. The upper curves in Fig. 5 are the plots of the function $\langle\mathbf{H}\rangle^{\prime}\left(\langle\mathbf{A}\rangle^{\prime}\right)$ when $T=60 \mathrm{keV}$ (lower full curve) and $T=150$ $\mathrm{keV}$ (upper full curve), respectively. The increase of $\langle\mathbf{H}\rangle^{\prime}$, when $T$ increases is transparent. The striking result is the apparent concavity of both curves. It is also found that the lower temperature, $60 \mathrm{keV}$, is low enough to allow $\langle\mathbf{H}\rangle^{\prime}$ to run, in practice, almost through the ground-state energies of both even and odd nuclei. As a check of our results, we repeated our calculations using the untuned energies, i.e., those which lack concavity. We then found that the low temperature limit of the $\langle\mathbf{H}\rangle$ curve went through the even nuclei only, namely, the limit curve follows the concave envelope of the experimental pattern.

For graphical and pedagogical convenience, Fig. 5 shows the plots for six nuclei only, but the same observations hold for full plots, with $110 \leq\langle\mathbf{A}\rangle^{\prime} \leq 134$. As a test, we also calculated $\langle\mathbf{H}\rangle^{\prime}$ and its low temperature limit when the levels of ${ }^{132} \mathrm{Sn}$ are omitted from the trace sum, Eq. (1). In that case, the concave envelope goes through the dots representing the two odd nuclei, ${ }^{131} \mathrm{Sn}$ and ${ }^{133} \mathrm{Sn}$, and ignores the dot representing ${ }^{132} \mathrm{Sn}$. Similar verifications of other concave envelopes were obtained by removing other nuclei.

To verify whether concave envelopes and concavity for $\langle\mathbf{H}\rangle^{\prime}$ result from negligible values of the entropy term in the free energy, or, more precisely, negligible values of its second derivative, a calculation of $T \mathbf{S}^{\prime}$ is in order. Figure 6 shows the difference, $T \mathbf{S}^{\prime}$, between the energy and the free energy, as a function of $\langle\mathbf{A}\rangle^{\prime}$, for $T=60$ and $150 \mathrm{keV}$, respectively. At the higher temperature, 150 $\mathrm{keV}$, wiggling effects seem to be small enough to allow for the concavity of $\langle\mathbf{H}\rangle^{\prime}$. At the lower temperature, $60 \mathrm{keV}$, the wiggling is stronger. As a consequence of such a strong wiggling, possibly translating into strong second derivatives, it 
is not excluded that SDs coming from $T \mathbf{S}^{\prime}$ might prevent $\langle\mathbf{H}\rangle^{\prime}$ from having the proven concavity property of the free energy. We must, therefore, numerically calculate second derivatives, see Eq. (10).

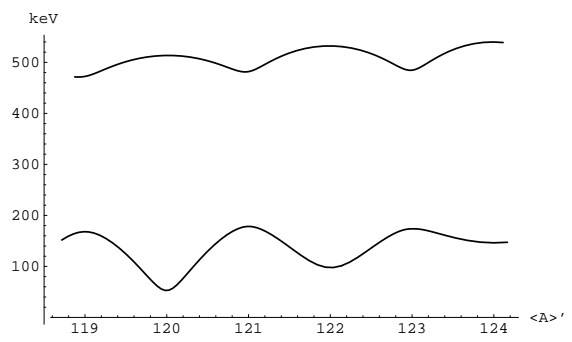

Figure 6: Entropy energy $T \mathbf{S}^{\prime}\left(\langle\mathbf{A}\rangle^{\prime}\right)$. Upper (lower) curve, $T=150$ (60) keV.

In Fig. 7 we show, for tuned data, plots of the rhs of Eq. (10), divided by $\left(\left\langle\mathbf{A}^{2}\right\rangle^{\prime}-\langle\mathbf{A}\rangle^{\prime 2}\right)^{3}$. This quantity must remain positive if $\langle\mathbf{H}\rangle^{\prime}$ shows concavity. The full line represents the situation when $T=60 \mathrm{keV}$, the dashed line corresponds to $T=150 \mathrm{keV}$. We see that the second derivative, $\partial^{2}\langle\mathbf{H}\rangle^{\prime} / \partial\langle\mathbf{A}\rangle^{\prime 2}$, remains positive almost always. For low temperatures, however, negative values may appear. For instance, the full curve, corresponding to $T=60 \mathrm{keV}$, indicates small, but definitely negative values around ${ }^{119} \mathrm{Sn}$. Numerical tests, which are not easy because of a difference effect between the two terms present in Eq. (10), show that the occurrence of such negative, actually moderate, values for lower temperatures might be somewhat frequent, while not systematic. Furthermore, such "negativity accidents" turn out to be worse when we use untuned data, maybe because the untuned data lack concavity in the first place.

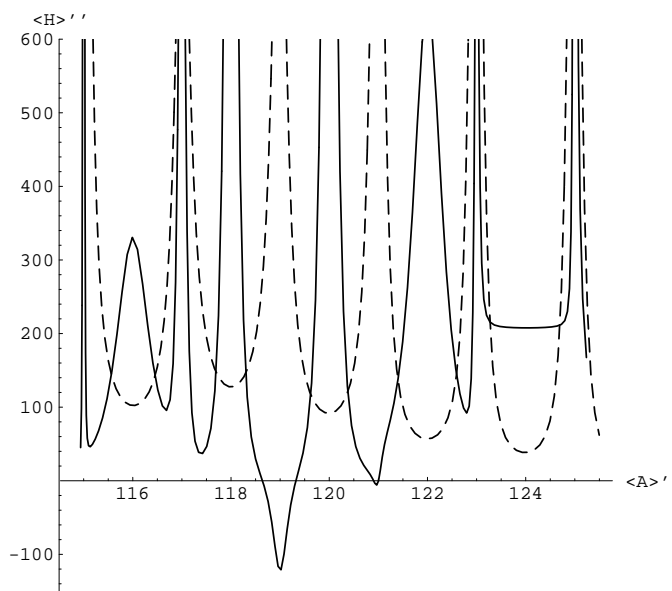

Figure 7: (a) Behavior of $H^{\prime \prime}=\partial^{2}\langle\mathbf{H}\rangle^{\prime} / \partial\langle\mathbf{A}\rangle^{\prime 2}$; full (dashed) curve, $T=60$ (150)keV. Notice tiny excursions in negative value territory by the low $T$ curve.

A likely reason for the negativity accidents with tuned data might be that the fluctuation of $\mathbf{A}$ is not large enough to justify our use of $\langle\mathbf{A}\rangle^{\prime}$ as a continuous 
variable. Since it interpolates between integers, a fluctuation of order $\sim 1$, or at least $\sim .5$, might be necessary. As shown by the plots in Fig. 8, corresponding to $T=60 \mathrm{keV}$ and $150 \mathrm{keV}$ for the lower and upper curves, respectively, a minimum temperature of order $\sim 150 \mathrm{keV}$ is needed to avoid too small a fluctuation of the particle number. Indeed, for, e.g. $\sim 100 \mathrm{keV}$, we found that the fluctuation is smaller than .5 for almost half of the calculated values of $\langle\mathbf{A}\rangle^{\prime}$.

At this stage, the situation can be summarized as follows. On the one hand, the tuned pattern of experimental energies shows concavity, but the concavity of $\langle\mathbf{H}\rangle^{\prime}$ as a function of $\langle\mathbf{A}\rangle^{\prime}$ is not sure, although it seems to occur most of the time. On the other hand, we have a theorem proving concavity for the free energy, either $\mathbf{F} \equiv\langle\mathbf{H}\rangle-T S=\mu\langle\mathbf{A}\rangle+\Omega$ or $\mathbf{F}^{\prime} \equiv\langle\mathbf{H}\rangle^{\prime}-T S^{\prime}=\mu\langle\mathbf{A}\rangle^{\prime}+\Omega^{\prime}$, as functions of $T$ and $\langle\mathbf{A}\rangle$ or $\langle\mathbf{A}\rangle^{\prime}$, respectively. For instance, elementary derivations show that, in that representation where $\langle\mathbf{A}\rangle$ (or $\left.\langle\mathbf{A}\rangle^{\prime}\right)$ and $\beta$ are the primary variables,

$$
\frac{\partial \mathbf{F}^{\prime}}{\partial \beta}=\beta^{-2} S^{\prime}, \quad \frac{\partial \mathbf{F}^{\prime}}{\partial\langle\mathbf{A}\rangle^{\prime}}=\mu \quad \text { and } \quad \frac{\partial^{2} \mathbf{F}^{\prime}}{\partial\langle\mathbf{A}\rangle^{\prime 2}}=\frac{T}{\left\langle\mathbf{A}^{2}\right\rangle^{\prime}-\langle\mathbf{A}\rangle^{\prime 2}} .
$$

To summarize this discussion, we see that the removal of the entropy term, leading from the free energy to just the energy, can sometimes destroy the concavity depending upon the temperature, but only weakly, e.g., see Fig. 7 .

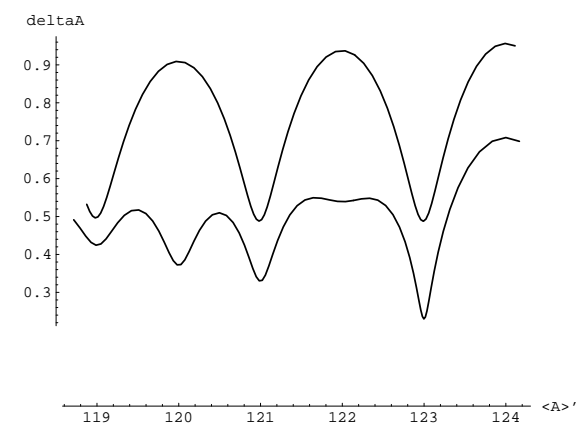

Figure 8: Particle number fluctuation. Upper (lower) curve, $T=150(60) \mathrm{keV}$.

A compromise may be found so that $T$ is low enough to make $T \mathbf{S}^{\prime}$ small with respect to $\mathbf{F}^{\prime}$ and high enough to ensure both sufficient values of $\Delta \mathbf{A}$ and a tolerable oscillating of $T \mathbf{S}^{\prime}$. Our rationale will be that $\langle\mathbf{H}\rangle^{\prime}$, even though it might deviate from concavity, will stay close enough to the concave $\mathbf{F}^{\prime}$. Their difference, $T \mathbf{S}^{\prime}$, a positive quantity, will define an error bar between a lower bound $\mathbf{F}^{\prime}$ and an upper bound $\langle\mathbf{H}\rangle^{\prime}$ for ground-state tuned energies. Consider again Fig. 5. There is no need to stress how, given $T$, the energy and free energy curves make a band defining upper and lower bounds for the experimental energies. Moreover, a similar, but narrower band is obtained if $T$ decreases. Figs. 5,6 and 8 suggests $T \sim 150 \mathrm{keV}$ for an error bar $\sim 500 \mathrm{keV}$ between energy and free energy, a smooth enough entropy energy and a sufficient fluctuation of the particle number. This order of magnitude for $T$ is compatible with the average 
level splitting, of order $\sim 250 \mathrm{keV}$, that is observed from the first 10 levels of ${ }^{110} \mathrm{Sn}, \ldots,{ }^{132} \mathrm{Sn}$ used in our calculations.

The following properties, i) the average energy and the free energy are increasing and decreasing, respectively, functions of increasing $T$, ii) the energy is larger than the free energy and iii) the entropy term by which they differ vanishes when $T$ vanishes, are not big surprises. It can be concluded that, in so far as thermodynamical functions can be calculated at low enough temperatures, precise "accuracy bands" may be available. Their, hopefully analytic, continuation for higher and/or lower values of $A$ than those available in nuclear tables provides a prediction scheme for exotic nuclei.

An estimate of the entropy term is now useful. Given $\mu$ and a large $\beta$, let $A_{0}$ and $E_{0}^{\prime}$ correspond to that nucleus whose ground-state energy maximizes the exponential, $\exp \left[\beta\left(\mu A-E_{0 A}^{\prime}\right)\right]$. Consider now the first subdominant exponential. It might be generated by the first excited state of the same nucleus, or by the ground-state of one of its neighbors. Let $A_{1}$ and $E_{1}^{\prime}$ be its parameters and define $\Delta=\mu\left(A_{1}-A_{0}\right)-\left(E_{1}^{\prime}-E_{0}^{\prime}\right)$. Concavity guarantees that $\Delta<0$. Whenever $\beta$ is large enough, it is trivial to reduce the grand canonical ensemble to a two state ensemble, and the entropy then boils down to $s=-e^{\beta \Delta} \beta \Delta$. Hence, the product, $T s=-e^{\beta \Delta} \Delta$, vanishes exponentially fast when $\beta \rightarrow \infty$. The rate of decrease is governed by that scale defined by $\Delta$, to be extracted from the tuned data. Then one can estimate an order of magnitude for the difference between the free energy and the energy. This estimate can be viewed as an error bar for the prediction of exotic nuclei via the present "concavity method".

We have applied the approach to several other isotopic regions, such as $\mathrm{Sm}, \mathrm{Hg}, \mathrm{Pb}$. In all cases, making the switch to the concave shape improves our ability to make extrapolations and interpolations for unknown bindings. However, estimating the free energy and the average value of $\mathbf{H}$, to set narrow upper and lower bounds on these binding energies, remains more difficult in drip line regions, because of the lack of experimental data on excited states in such regions. Very simple linear extrapolations still provide, nonetheless, error bars with an order of magnitude $\Delta E \sim \pm 400 \mathrm{keV}$, similar to the best "band" seen in Fig. 5. A few examples of the kind of results that we can obtain from this thermodynamical error-bar analysis, when using the method described in Sec. 2, are these conservative estimates of the bindings of ${ }^{171} \mathrm{Hg},{ }^{208} \mathrm{Hg}$ and ${ }^{209} \mathrm{Hg}$, namely $1314.33 \mathrm{MeV} \pm 400 \mathrm{keV}, 1629.13 \mathrm{MeV} \pm 400 \mathrm{keV}$ and 1632.16 $\mathrm{MeV} \pm 400 \mathrm{keV}$, respectively.

\section{Two-dimensional analysis; density functional}

We now want to consider binding-energy systematics in two dimensions, i.e., $\mathrm{N}$ and Z. The second differences studied in this Section are, $S D_{n}=E_{N+1, Z}+$ $E_{N-1, Z}-2 E_{N Z}, S D_{p}=E_{N, Z+1}+E_{N, Z-1}-2 E_{N Z}, S D_{b}=\left(E_{N+1, Z+1}+\right.$ $\left.E_{N-1, Z-1}\right) / 2-E_{N Z}$ and $S D_{s}=\left(E_{N+1, Z-1}+E_{N-1, Z+1}\right) / 2-E_{N Z}$. If $N$ and $Z$ were continuous, and, furthermore, if $E_{N Z}$ were a smooth function of $N$ and $Z$, the numbers, $S D_{n}, S D_{p}, S D_{b}$ and $S D_{s}$, might be interpreted as estimates 
of second derivatives of the function $E(N, Z)$ in the neutron, proton, constant $N-Z$ and constant $A$ directions, respectively. Their connections with neutron, proton and p-n pairings and with a symmetry energy are also transparent.

Setting aside what we consider to be "light" nuclei, namely those of the s-, p-, sd- and $f_{7 / 2}$ shells, we calculated these four SDs for the rest of the known nuclear table. Their maxima and minima are found to be of order $\sim\{4840,6180,4530,5900,-3670,-3080,-3160,-2150\} \mathrm{keV}$, respectively. The lhs parts of Figs. 9-12 show, in scattered plots with respect to $A$, the patterns of those SD values. The negative SDs, contradicting concavity, are due mainly to pairing effects. An extension of the correction done in Sec. 2 is in order. Let $p$ be a mass dependent [10] estimate of pairing. Diminish the binding of doubly even nuclei by $p$, increase the binding of doubly odd ones by $p$, and leave odd nuclei untouched. We set, empirically, $p=1160-A$. There is no doubt that better parametrizations of $p$ are possible [10, but the present linear decrease suffices for our demonstration. With this correction, the worst SDs become $\sim\{2790,3970,2470,3680,-1500,-930,-980,-120\} \mathrm{keV}$, respectively. As expected, this is a large reduction of both positive and negative amplitudes. This is shown by the rhs parts of Figs. 9-12, respectively.
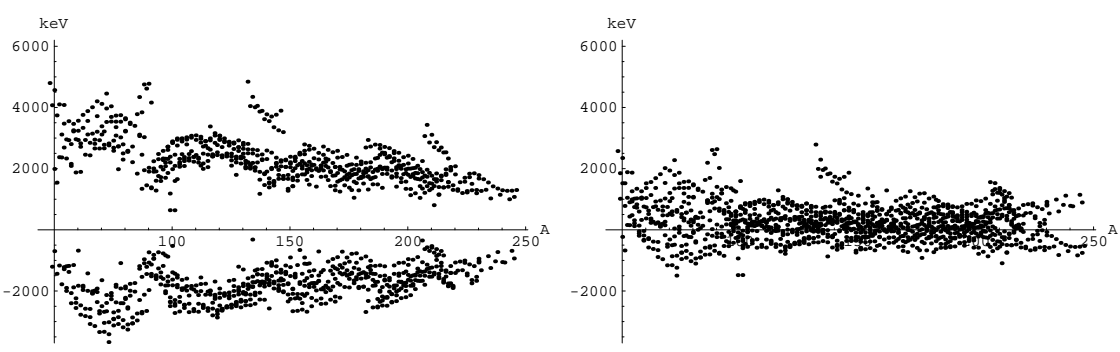

Figure 9: Scatter plot of convex second differences $S D_{n}$ in the neutron direction throughout the table of known nuclei for $27<A<251$. Left: with bare energies. Right: after a pairing correction.
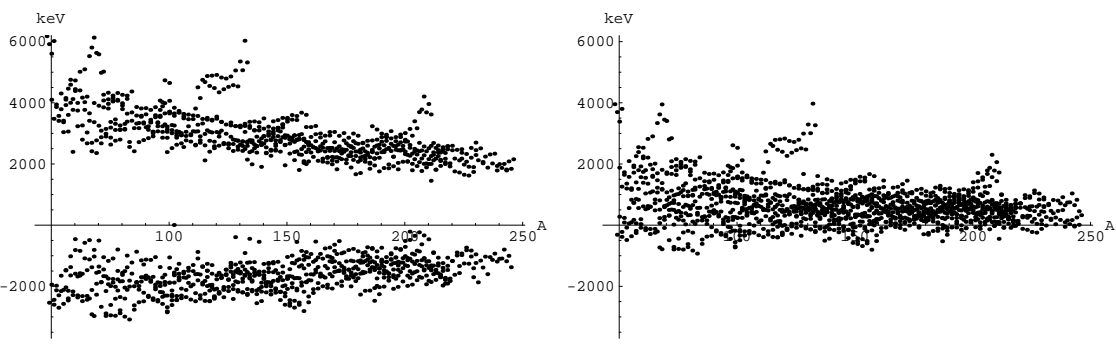

Figure 10: Same as Fig. 9, but for $S D_{p}$ in the proton direction.

While the pairing correction has not completely suppressed negative SDs, 
note the worst minimum is still of order $\sim-1500 \mathrm{keV}$, concavity has been improved significantly and extrapolations are likely made more secure.

Similar to our one-dimensional analysis in Sec. 2, one can now add to the energies a paraboloid term, of the form, $u\left(N-N_{0}\right)^{2}+2 v\left(N-N_{0}\right)\left(Z-Z_{0}\right)+$ $w\left(Z-Z_{0}\right)^{2}$, in order to get rid of the residual, negative SDs. (We recall that SDs do not depend on $N_{0}$ and $Z_{0}$, which are only parameters for graphical and/or numerical convenience.) Notice from Fig. 12, and the corresponding worse minimum, of order $\sim-120 \mathrm{keV}$, that the direction where $A$ is constant does not deviate much from concavity. The dominant term in the paraboloid must, therefore, indicate a direction approximately orthogonal, and also take into account the bend of the global stability valley towards neutron excess.
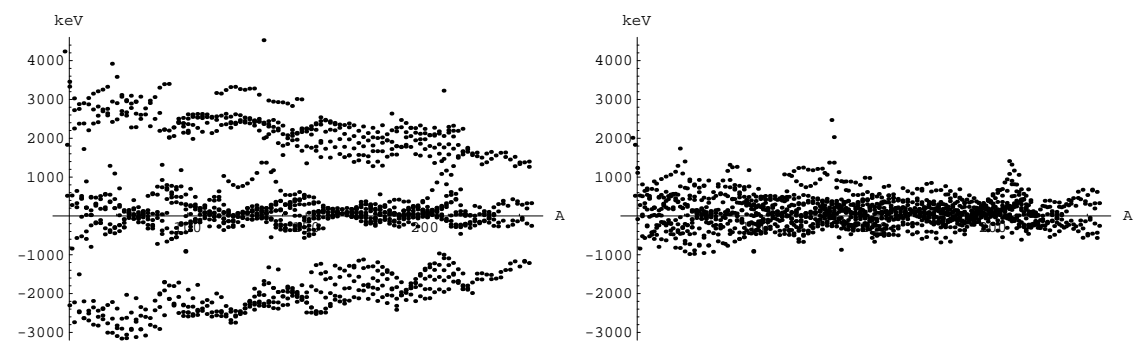

Figure 11: Same as Fig. 9, but for $S D_{b}$ in the constant $N-Z$ direction.
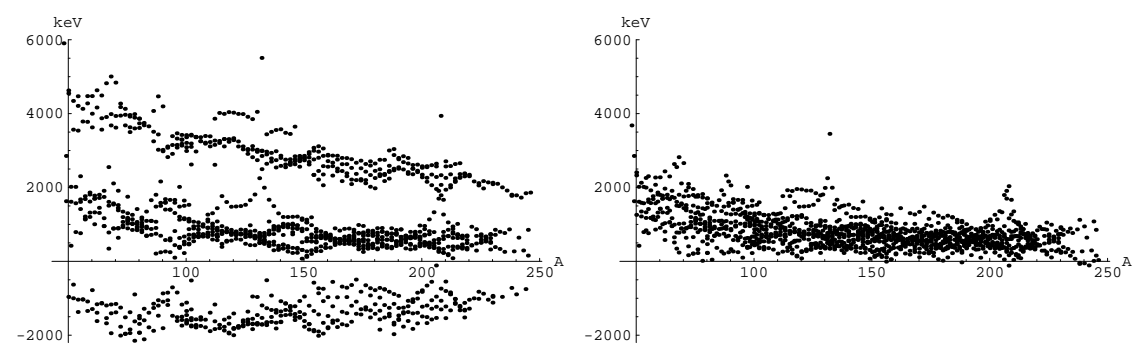

Figure 12: Same as Fig. 9, but for $S D_{s}$ in the direction where $A$ is constant.

There is, furthermore, no doubt that the two-dimensional free energy, now a function of both $N$ and $Z$ made continuous by a grand canonical ensemble, remains a concave function, available for lower bounds. This is a theorem, which can be proven in a straightforward manner following the previous derivation for one dimension. Simultaneously, it is likely that the mean energy will be again "almost concave", to yield upper bounds, in which case the same error bar that was estimated in Sec. 3 remains valid.

A systematic use of such properties throughout the nuclear table is way beyond the scope of the present paper. Rather, we want to discuss the relation of concavity with the derivation of a nuclear density functional. Recall that, 
given an arbitrary many-body density matrix $\mathcal{M}$ in Fock space, the density functional derives from the definition [11,

$$
\mathcal{F}\left[\rho_{p}, \rho_{n}\right]=\operatorname{Inf}_{\mathcal{M} \rightarrow \rho_{p}, \rho_{n}} \operatorname{Tr} \mathbf{H} \mathcal{M} .
$$

Here the symbol, $\mathcal{M} \rightarrow \rho_{n}, \rho_{p}$, means that a minimization of the energy is performed upon density matrices having the same neutron and proton profiles $\rho_{n}, \rho_{p}$. A nucleus is identified by the integrals, $N=\int d \vec{r} \rho_{n}(\vec{r})$ and $Z=$ $\int d \vec{r} \rho_{p}(\vec{r})$, and a later minimization is performed with respect to the profiles $\rho_{n}, \rho_{p}$ under such identification constraints,

$$
E_{Z N}=\operatorname{Inf}_{\rho_{n} \rightarrow N, \rho_{p} \rightarrow Z} \mathcal{F}\left[\rho_{n}, \rho_{p}\right] .
$$

The functional $\mathcal{F}$ should be universal, in the nuclear physics sense. Namely, it should not depend on $N$ and $Z$. (In atomic and molecular physics, universality of the DF has a different meaning; it refers to arbitrary external potentials.) While BCS and Hartree-Bogoliubov calculations allow a distinction between even and odd particle numbers, universality in density profile space does not allow such a separate treatment. Observe, furthermore, that, because of the fact that there are many convexities in the pattern of raw energies, there exist many cases where, for instance, three nuclei, $A_{1}, A_{2}, A_{3}$, are such that $N_{2}=\left(N_{1}+N_{3}\right) / 2$ and $Z_{2}=\left(Z_{1}+Z_{3}\right) / 2$ and $\left(E_{1}+E_{3}\right) / 2<E_{2}$. Then, in a search for $E_{2}$, the mixture density matrix, $\mathcal{M}_{\text {mix }}=\left(\left|A_{1}\right\rangle\left\langle A_{1}|+| A_{3}\right\rangle\left\langle A_{3}\right|\right) / 2$, provides the correct average particle numbers $N_{2}, Z_{2}$, but an absurd energy, lower than $E_{2}$. Concavity is a mandatory condition for the universality of $\mathcal{F}$.

One way to simultaneously implement pairing corrections and use a minimal paraboloid term consists in adding to the physical $\mathbf{H}$ terms such as, $p \times$ $\left[\cos ^{2}(\pi \mathbf{N} / 2)+\cos ^{2}(\pi \mathbf{Z} / 2)-1\right]+u \mathbf{N}^{2}+2 v \mathbf{N} \mathbf{Z}+w \mathbf{Z}^{2}$. (Notice how the cosines take advantage of the continuous nature of $\langle\mathbf{N}\rangle$ and $\langle\mathbf{Z}\rangle$ at finite temperatures.) Concavity is achieved if $u, v, w$ are tuned to compensate for the worst tuned SD minima listed above, namely $\{-1500,-930,-980,-120\}$. A rough solution consists in taking $p \sim 1100$ and a "circular" ansatz, $P \times\left(\mathbf{N}^{2}+\mathbf{Z}^{2}\right)$, with $P \sim 800$, but less brutal solutions are obviously worthy of consideration, in order to minimize this tampering with the Hamiltonian. In any case, we stress

that the operators, $\mathbf{N}$ and $\mathbf{Z}$, commute with $\mathbf{H}$, and, therefore, that the present counterterms do not perturb nuclear dynamics. Once again we emphasize that this approach unifies the treatment of odd and even nuclei. The fact that the nuclear density functional needs to originate from a Hamiltonian, completed by such counterterms, illustrates how important concavity is for making predictions for nuclei far from stability, i.e., for the so-called exotic nuclei.

\section{Summary, discussion and conclusion}

We have demonstrated how a list of ground-state energies for a sequence of isotopes can be turned into a concave pattern. This involves simple manipulations; for instance, an explicit term, accounting for pairing in even nuclei, can 
be subtracted from the bindings. This unifies the treatment of odd and even nuclei, a notoriously difficult problem. If needed, a small quadratic correction can also be added to guarantee concavity at all points along the sequence.

Similar arguments leading to concavity clearly hold for isotones as well, and, furthermore, for any other sequence of neighboring nuclei in any direction across the nuclear table. Once this empirical tuning has been implemented, linear (or more general) extra- and interpolations of the concave pattern can provide surprisingly accurate and robust estimates of, or bounds for, binding energies. These tuning terms, which are added to induce concavity, are, of course, subtracted in fine.

This work then defined a more ambitious extra- and interpolation scheme, involving thermodynamical functions from a grand canonical ensemble, because such functions may have rigorous concavity properties. Theorems are, indeed, available to prove such properties. For instance, the free energy is a concave function of the average particle number and is also a decreasing function of the temperature. We also found strong numerical evidences concerning the average energy. This average energy at nonzero temperature turns out, in general, to be a concave function of the average particle number, except for "minor accidents," which depend upon the temperature.

For every given, finite temperature, we found that the average energy and the free energy, as functions of the average particle number, give upper and lower bounds, respectively, for the concave envelope of the ground-state energies. When the temperature vanishes, both bounds converge to the exact results. At this vanishing temperature, however, the analyticity of such thermodynamical functions is lost, because their limit is only piecewise continuous. It is, therefore, necessary to retain a minimum temperature if one wants to obtain practical extrapolations for the prediction of exotic nuclei. A minimum amount of particle number fluctuation is necessary to validate the conversion of particle number, an initially discrete quantity, into a continuous variable.

We, therefore, implemented numerical estimates of several thermodynamical functions at moderate temperatures, a few hundred $\mathrm{keV}$ at most. This yields a first result, namely a "band", enclosing ground-state energies between the average energy and the free energy. The width of the band defines an error bar which can be trusted when extrapolations are made.

A difficulty arises, however, because of an insufficient number of excited states for nuclei at both ends of any sequence of isotopes. These excited-state energies are essential for calculating a meaningful value of the average energy for any value of A. More often, only the ground-state energy is known for such neutron very rich or neutron very poor nuclei. The calculation of the average and free energies is, thus, possible only in an interval smaller than the interval of masses where ground states are known. Two tactics are then available, namely i) an extrapolation only of the sequence of ground-state energies and ii) an extrapolation of the thermodynamical functions, starting from a smaller interval. We found that the first tactic, namely at zero temperature, is reliable, especially when completed by the error bar derived from the finite temperature considerations. This work also tested the second tactic, with some success but 
with the limitation due to the lack of known excited states. The lack leads to edge effects in the calculation of thermodynamical functions. Therefore, if linear, or polynomial, or more general analytical fits of such thermodynamical functions are attempted, it is better to restrict them from a slightly smaller mass interval. Then, extrapolations should be reliable within one and maybe two mass units towards drip lines.

We can make the strong conclusion that the combination of concavity and extrapolations of thermodynamical functions gives a systematic set of upper and lower bounds for the prediction of ground-state energies.

Our last, and perhaps most important result, is the connection between concavity and the universality of the density functional. In a two-dimensional analysis, we showed that a paraboloid term with respect to the proton and neutron number operators must be added to the Hamiltonian, so as to guarantee obtaining consistent energy minima everywhere in density space. This term can be made minimal if counterterms for pairing are also added.

Acknowledgements: We thank I. Allison for helpful discussions and assistance with the management of the data sets. It is a pleasure for B. R. B. and B. G. G. to thank TRIUMF, Vancouver, B. C., Canada, where part of this work was done, for its hospitality. The Natural Science and Engineering Research Council of Canada is thanked for financial support. TRIUMF receives federal funding via a contribution agreement through the National Research Council of Canada. B. R. B. also thanks Institut de Physique Théorique, Saclay, France, for its hospitality, where another part of this work was carried out, and the Gesellschaft für Schwerionenforschung (GSI), Darmstadt, Germany, for its hospitality during the preparation of this manuscript, and acknowledges partial support from NSF grant PHY0555396 and from the Alexander von Humboldt Stiftung.

\section{References}

[1] C.F. von Weizsäcker, Z. Physik 96, 431 (1935).

[2] R.B. Cakirli, D.S. Brenner, R.F. Casten and E.A. Millman, Phys. Rev. Lett. 94, 092501 (2005).

[3] UNEDF SciDAC Collaboration, www.unedf.org/

[4] G. Audi, A.H. Wapstra and C. Thibault, Nucl. Phys. A 729, 337 (2003)

[5] G. Audi and A. H. Wapstra, Nucl. Phys. A 595, 409 (1995).

[6] G. Audi, O. Bersillon, J. Blachot and A. H. Wapstra, Nucl. Phys. A 624, 1 (1997).

[7] P. Ekström, Nuclear Structure and Decay Data, http://nucleardata.nuclear.lu.se/database/masses/

[8] Table of Isotopes, Firestone, Shirley, Baglin, Chu, Zipkin eds, Wiley 
[9] http://www.nndc.bnl.gov/nudat2/

[10] J.M. Eisenberg and W. Greiner, Nuclear Models (North Holland/American Elsevier, Amsterdam, 1975); K.S. Krane, Introductory Nuclear Physics, (Wiley, New York, 1988); C.H. Dasso and M.J. Gallardo, Phys. Rev. C 74, 014307 (2006)

[11] M. Levy, PNAS 766062 (1979); E.H. Lieb, Int. J. Quant. Chem. 24, 243 (1983) 